\title{
Preliminary colonoscopy facilitates retrograde double-balloon enteroscopy
}

Authors

Institution
Samuel Han, Kanishka Bhattacharya, David R Cave

Department of Internal Medicine, University of Massachusetts Medical School, Worcester, Massachusetts 01655 USA submitted 31. January 2014 accepted after revision 7. July 2014

\section{Bibliography}

Dol http://dx.doi.org/ 10.1055/s-0034-1377760 Published online: 26.9.2014 Endoscopy International Open 2014; 02: E241-E243

(c) Georg Thieme Verlag KG Stuttgart · New York E-ISSN 2196-9736

\section{Corresponding author}

\section{Samuel Han, MD}

UMass Memorial Medical Center 55 Lake Avenue North

Worcester, Massachusetts 01655 USA

Fax: +01-508-856-3981 Samuel.Han@umassmemorial. org
Background and study aims: Retrograde doubleballoon enteroscopy (RDBE) has a high failure rate due to difficulty intubating the ileo-cecal (IC) valve. We examined the utility of a pre-RDBE colonoscopy using a pediatric colonoscope to clean the cecum and perform an initial intubation of the IC valve.

Patients and methods: This study is a retrospective review of RDBE procedures for 45 patients at a single tertiary-care center to examine the success of IC intubation, maximal depth of enteroscope insertion, and duration of the procedure.
Results: The IC intubation success rate among patients who underwent RDBE using this novel method was $100 \%$ as compared to $72.7 \%$ using the traditional method $(P<0.003)$.

Conclusions: RDBE preceded by colonoscopy had a significantly higher IC intubation success rate, compared to RDBEs performed using the traditional method. Results support the use of this novel method when IC valve intubation using standard methods is difficult, and it may limit the need for repeat procedures or the use of other modalities for examining the small bowel.

\section{Abbreviations \\ $\checkmark$ \\ BMI Body mass index \\ DBE Double-balloon enteroscopy \\ IC Ileo-cecal \\ RDBE Retrograde double-balloon enteroscopy \\ TI Terminal ileum}

\section{Introduction \\ $\nabla$}

Double-balloon enteroscopy (DBE), first described by Yamamoto et al [1], allows examination of the small intestine, and retrograde DBE (RDBE) permits access to the distal small intestine. The success rates of RDBE procedures are variable. Mehdizadeh et al [2] found a 79\% success rate, defining success as intubation of the IC valve, in their review. May et al [3], however, failed to reach the terminal ileum (TI) in $7 \%$ of patients while Ell et al [4] and Heine et al [5] reported $14 \%$ and $58 \%$ failure rates, respectively. The highest success rate was achieved by the group with the most experience, Yamamoto et al [6], who successfully intubated the IC valve in all of their 89 patients who underwent RDBE.

Intubation of the IC valve via the retrograde approach remains challenging. Mehidizadeh et al
[7] found no significant improvement in the endoscopist's performance with increased experience, and found only a significant decline in overall procedure and fluoroscopy times. While DBE has been shown to facilitate difficult colonoscopies [8], we noted that performing a pre-DBE colonoscopy using a pediatric colonoscope improved RDBE IC intubation rates. The rationale for such a procedure is that conventional colonoscopy is quick and the more rigid colonoscope facilitates the initial IC valve intubation. This dilates and orients the valve, enabling access with the more flexible DBE. We therefore examined the IC intubation rates, procedure duration, and maximal depth of enteroscope insertion among those patients who received a pre-colonoscopy followed by RDBE and compared the results with those who received a conventional RDBE.

\section{Patients and methods \\ $\nabla$}

This study was approved by the institution's Committee for the Protection of Human Subjects in Research. A computer program randomly chose 45 patients from a pool of all patients who underwent RDBE procedures during 2011-2013. All procedures were performed by either of two ex- 
perienced endoscopists, who had both performed approximately 50 RDBEs prior to the study period, and a gastroenterology fellow. Consent was obtained for all procedures and all patients underwent a bowel-cleansing regimen consisting of four liters of polyethylene glycol and overnight fasting. The Fujinon doubleballoon enteroscopy system (Fujinon EN-450T5 enteroscope; Jujifilm Medical Systems, Wayne, New Jersey, USA) was used in all procedures and the pediatric colonoscope (Olympus PCF 160 or 180; Olympus America, Inc., Melville, New York, USA) was utilized in those procedures involving a pre-RDBE colonoscopy. Sedation was achieved by either conscious sedation with fentanyl and midazolam or with general anesthesia at the discretion of the endoscopist in conjunction with the anesthesiologist, depending on the cardiovascular and respiratory status of the patient.

In patients who received a pre-RDBE colonoscopy, a pediatric colonoscope was introduced through the anus and advanced into the ileum. The colonoscope allowed easy cleaning of the cecum, and dilation and orientation of the ileo-cecal (IC) valve. After advancing approximately $20 \mathrm{~cm}$ into the ileum, which took 5-15 minutes, the colonoscope was removed and the standard RDBE method was followed as described by Yamamoto et al [1]. Deeper intubation of the ileum by colonoscopy or by RDBE was facilitated by placing the patient supine.

Successful enteroscope intubation consisted of reaching the TI. Stability of enteroscope intubation was deemed as reaching 15 $20 \mathrm{~cm}$ proximal to the IC valve. The duration of the procedure was defined as the time of endoscope insertion (including the preRDBE colonoscope) to the time of removal of the enteroscope. The maximal depth of insertion was defined as the distance encompassed by the enteroscope until advancement was no longer possible.

Demographic data, including age, gender, height, weight, indications for RDBE, sedation method, procedure duration, interventions, and immediate adverse effects were electronically documented at the time of the procedure. Patient's prior medical and surgical history were reviewed.

\section{Statistical analysis}

The mean, standard deviation (SD), and range were calculated for the continuous data obtained for each patient. Comparisons between the two groups were carried out using the Mann-Whitney $U$ test. A $P$ value of $<0.05$ was considered to be statistically significant. All statistical analysis was performed using the SPSS statistical software package [9].

\section{Results}

$\nabla$

\section{Demographics}

Retrograde RDBEs (45 patients) performed from 2011-2013 were randomly chosen for this study. Twenty-three of the patients were women (51.1\%) and twenty-two of them were men (48.9\%). The mean (SD) patient age was 59.7 (16.0) years (range $22-84)$. The mean (SD) patient body mass index (BMI) was 30.1 (7.33) $\mathrm{kg} / \mathrm{m}^{2}$ (range $16.2-52.4$ ) and 12 of 45 patients $(26.7 \%$ ) had a BMI over 30. The main indications for the procedure were occult gastrointestinal bleeding (50\%), masses (26\%), video capsule endoscopies with abnormal findings (11\%), and iron-deficiency anemia (8\%). In comparing the results of the group that received the pre-RDBE colonoscopy with those of the group that received the traditional method, the only statistically significant difference was within the gender. The group that underwent the novel procedure had fifteen men and eight women, while the group that underwent the traditional procedure had seven men and fifteen women $(P<0.0004)$.

\section{Procedure results}

The pre-RDBE colonoscopy method was used in 23 patients $(51.1 \%)$ and the standard DBE method was used in 22 patients (48.9\%). Among the patients who received the pre-RDBE colonoscopy, successful, stable intubation of the IC valve was achieved in all 23 patients (100\%), compared to only $16 / 22(72.7 \%)$ of those who underwent the traditional procedure $(P<0.003)$. The mean (SD) of maximal depth of insertion was $78.2(18.1) \mathrm{cm}$ and $67.2(17.1)$ $\mathrm{cm}$ in the novel procedure group and the traditional procedure group, respectively, which were not significantly different $(P<$ 0.65 ). In addition, the mean (SD) for the duration of the procedure in the novel and the traditional procedure groups were 81.6 (8.9) minutes and 95 (13.2) minutes, respectively. These results were not significantly different $(P<0.53)$. Successful diagnosis and intervention (identification and treatment of bleeding source or lesion) was performed in $13 / 23(56.5 \%)$ in the novel procedure group and $7 / 22$ (32\%) in the traditional procedure group, and these results were not significantly different $(P<0.09)$. The novel procedure group and the traditional procedure group had $3 / 23$ patients (13.0\%) and $3 / 22$ patients (13.6\%) with poor bowel preparation, respectively. One of the three patients in the latter group experienced an unsuccessful IC intubation. The two endoscopists performed 25 and 20 RDBEs, and the failure rates when they used the traditional method were $3 / 13(23.1 \%)$ and $3 / 9$ (33.3\%), respectively $(P<0.01)$. Intubation rates for men $(90.9 \%)$ and women (82.6\%) were not significantly different $(P<0.41)$.

\section{Discussion}

Retrograde DBE poses a technical challenge to the endoscopist and requires a significant learning curve [7]. Thorough and consistent training under thoughtful mentorship is crucial to not only intubate the IC valve, but also to avoid non-spiral looping and enteroscope withdrawal upon engagement of the ileum. Consistent with this challenge, various groups have reported unsuccessful IC valve intubation rates ranging from 7\%-58\%. The difficulty in successful intubation occurs when the valve orifice cannot be oriented appropriately or the valve itself cannot be intubated [10]. By performing a pre-RDBE colonoscopy, which is usually a quick procedure, the more rigid pediatric colonoscope allowed for easier intubation by dilating and orienting the valve to later facilitate IC intubation with the more flexible DBE. In addition, although not quantified, using the colonoscope allowed easier cleaning of the residual luminal contents from the cecum due to its suction channel, which is larger than that of the DBE. Rapid withdrawal of the colonoscope left a straightened colon that facilitated rapid insertion of the DBE.

Retrograde DBE utilizing this novel method of performing a colonoscopy before the RDBE had a significantly higher IC intubation success rate, compared to RDBEs using the traditional method. Within the traditional method group, a similar success rate to that of the Mehdizadeh et al [2] group was found $(72.7 \%$ vs $79 \%)$. However, with the novel method, we achieved successful intubation in $100 \%$ of our patients. While not statistically significant, the mean procedure time was shorter on average, despite the additional colonoscopy. 
The main limitation of this study is that it is a small, retrospective, single-center study. Multi-center prospective studies are needed to compare the results of this novel method with those obtained with the traditional method. The gender disparity between the two groups does present a potential confounding variable; there were fifteen women in the traditional procedure group and only eight women in the novel procedure group. However, there was no significant association between gender and successful intubation rates. Alternative techniques to improve IC valve intubation have been described, including a study by Ross et al [11] in which a balloon was inflated over a guidewire passed through the IC valve, and Despott et al [10] in which the enteroscope balloon was inflated in the TI while the enteroscope and overtube were pulled back to straighten the ileo-colonic angle. The study reported herein offers another option the endoscopist may use when confronted with a difficult RDBE intubation.

Competing interests: Dr. Cave and Dr. Bhattacharya are consultants for Olympus America.

\section{References}

1 Yamamoto H, Sekine $Y$, Sato $Y$ et al. Total enteroscopy with a nonsurgical steerable double-balloon method. Gastrointest Endosc 2001; 53: 216-220

2 Mehdizadeh S, Han NJ, Cheng DW et al. Success rate of retrograde double-balloon enteroscopy. Gastrointest Endosc 2007; 65: 633-639

3 May A, Nachbar L, Ell C. Double-balloon enteroscopy (push-and-pull enteroscopy) of the small bowel: feasibility and diagnostic and therapeutic yield in patients with suspected small bowel disease. Gastrointest Endosc 2005; 62: $62-70$

4 Ell C, May A, Nachbar L et al. Push-and-pull enteroscopy in the small bowel using the double-balloon technique: results of a prospective European multicenter study. Endoscopy 2005; 37: 613-616

5 Heine GDN, Hadithi M, Groenen MJM et al. Double-balloon enteroscopy: indications, diagnostic yield, and complication in a series of 275 patients with suspected small-bowel disease. Endoscopy 2006; 38 : $42-48$

6 Yamamoto H, Kita H, Sunada K et al. Clinical outcomes of double-balloon endoscopy for the diagnosis and treatment of small-intestinal diseases. Clin Gastroenterol Hepatol 2004; 2: 1010-1016

7 Mehdizadeh S, Ross A, Gerson L et al. What is the learning curve associated with double-balloon enteroscopy? Technical details and early experience in 6 US tertiary care centers Gastrointest Endos 2006; 64: $740-750$

8 Gay G, Delvaux M. Double-balloon colonoscopy after failed colonoscopy: a pilot series with a new instrument. Endoscopy 2007; 39: 788 792

9 SPSS Version 15.0 for Windows. Chicago: SPSS Inc; 2007

10 Despott E, Fraser C. Achieving successful ileal intubation during retrograde double balloon enteroscopy: description of a novel, alternative technique. Endoscopy 2009; 41: E309-E310

11 Ross A, Waxman I, Semrad C et al. Balloon-assisted intubation of the ileocecal valve to facilitate retrograde double balloon enteroscopy. Gastrointest Endosc 2005; 62: 987-988 